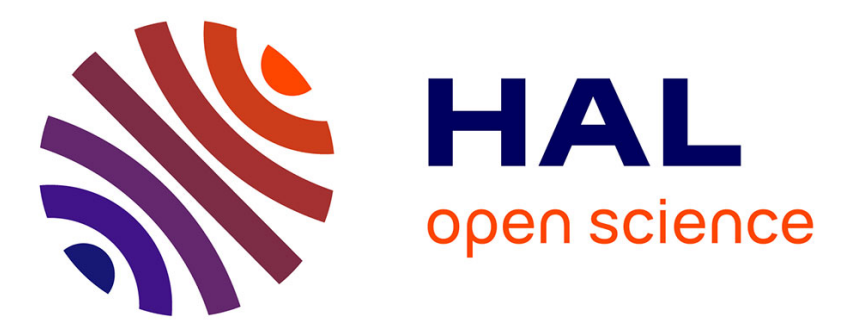

\title{
Direct Observation of the Two Lowest Exciton Zero-Phonon Lines in Single CdSe/ZnS Nanocrystals
}

Louis Biadala, Yann Louyer, Philippe Tamarat, Brahim Lounis

\section{To cite this version:}

Louis Biadala, Yann Louyer, Philippe Tamarat, Brahim Lounis. Direct Observation of the Two Lowest Exciton Zero-Phonon Lines in Single CdSe/ZnS Nanocrystals. Physical Review Letters, 2009, 103, pp.037404. 10.1103/PhysRevLett.103.037404 . hal-00611545

\section{HAL Id: hal-00611545 \\ https://hal.science/hal-00611545}

Submitted on 26 Jul 2011

HAL is a multi-disciplinary open access archive for the deposit and dissemination of scientific research documents, whether they are published or not. The documents may come from teaching and research institutions in France or abroad, or from public or private research centers.
L'archive ouverte pluridisciplinaire HAL, est destinée au dépôt et à la diffusion de documents scientifiques de niveau recherche, publiés ou non, émanant des établissements d'enseignement et de recherche français ou étrangers, des laboratoires publics ou privés. 


\title{
Direct observation of the two Lowest Exciton Zero-Phonon Lines in Single CdSe/ZnS Nanocrystals
}

\author{
L. Biadala, Y. Louyer, Ph. Tamarat and B. Lounis* \\ ${ }^{1}$ Centre de Physique Moléculaire Optique et Hertzienne, Université de Bordeaux and CNRS, \\ 351 cours de la Libération, Talence, F-33405 (France)
}

We report a spectroscopic study of highly photostable individual CdSe/ZnS colloidal nanocrystals. At low temperature, photoluminescence spectra display two sharp zero-phonon lines which we attribute to the radiative recombination from the two lowest levels of the bandedge exciton fine structure. For the first time, resonant photoluminescence excitation spectra of these lines is performed, and spectral diffusion broadening of $10 \mu \mathrm{eV}$ is measured over integration times of $100 \mathrm{~ms}$, corresponding to an optical coherence lifetime longer than 100 ps.

*b.lounis@cpmoh.u-bordeaux1.fr 
Over the past decade, interest in low-dimensional, mesoscopic systems, such as semiconductor nanocrystals (NCs), has grown dramatically [1]. These NCs bridge the gap between single molecules and the solid state, thereby offering the opportunity to study the evolution of bulk properties. Additionally, their size-dependent optical properties due to quantum confinement make them ideal candidates for tunable absorbers and emitters in applications such as nanoscale electronics [2-4], laser technology [5] and biological fluorescent labelling [6, 7]. Chemically synthesized CdSe NCs are interesting for many of these applications due to their bright luminescence, which is size tunable across the visible spectrum [8].

A detailed understanding of the band-edge exciton fine structure is crucial for these applications. It is well established, on both theoretical and experimental grounds [9], that in spherical or nearly spherical colloidal CdSe NCs the band-edge exciton $\left(1 S_{3 / 2}-1 S_{e}\right)$ is split by the intrinsic electron-hole exchange interaction and by the crystal field of the hexagonal lattice into five energy levels $[10,11]$. The exciton ground state $|F\rangle$, which is often referred to as the dark exciton, has a total spin projection on the crystal hexagonal axis $J= \pm 2$. It is located a few meV below the lowest-energy optically active $\left(J= \pm 1^{L}\right)$ exciton state $|A\rangle$. Studies of the photoluminescence (PL) spectra and decay as a function of temperature and external magnetic fields $[10,12-15]$ showed that the luminescence arises from the two thermally mixed lowest states of the band-edge exciton fine structure. At liquid helium temperatures, the luminescence of NCs with strong confinement (radius smaller than $3 \mathrm{~nm}$ ) originates primarily from the long-lived (hundreds of nanoseconds) dark exciton state, because the bright-to-dark spin-flip rate is much larger than the radiative recombination rate of the bright exciton state $[10,14,16]$. The PL spectra display a single zero-phonon line (ZPL) [17-19] which is therefore attributed to the weakly allowed radiative recombination of the dark state.

In this work, we report a $\mathrm{cw}$ and time resolved spectroscopic study of individual CdSe NCs with a weaker exciton confinement (radius of $\sim 4 \mathrm{~nm}$ ). At low temperature, these NCs display emission spectra with two sharp ZPLs that we attribute to the radiative recombinations from the two lowest exciton levels (bright and dark), as a consequence of a weak spin-flip rate between these two states. We also performed, for the first time, resonant photoluminescence excitation of these transitions and obtained excitation lines as narrow as $10 \mu \mathrm{eV}$ over an integration time of $100 \mathrm{~ms}$. 
Samples of CdSe/ZnS core-shell NCs (Qdot655 streptavidin conjugates from Quantum Dot Corp., peak emission at $655 \mathrm{~nm}$ at room temperature) are prepared by spin coating clean glass coverslips with a nanomolar solution of NCs in polyvinyl alcohol. A homebuilt scanning confocal microscope is used to image single NCs excited with the $532 \mathrm{~nm}$ line of a cw frequency doubled Nd:YAG laser. It is based on a 0.95 NA objective which is inserted in a helium cryostat together with the sample and a piezo-scanner. The emitted photons are filtered from the scattered excitation light by a bandpass filter ( $60 \mathrm{~nm}$ FWHM) and sent to a single-photon-counting avalanche photodiode and a spectrometer (1800 lines/mm grating) equipped with a liquid-nitrogen-cooled camera. The PL decay measurements are performed with a conventional time correlated single photon counting setup, using a pulsed laser source (optical parametric oscillator at $570 \mathrm{~nm}, 250$ fs pulse width, $2.5 \mathrm{MHz}$ repetition rate). The instrumental response function of the system was 300 ps FWHM. The high stability of our experimental microscopy setup allows the study of the same single NC over a wide range of temperatures and over several days.

At $2 \mathrm{~K}$ the luminescence intensity of most of the single NCs is remarkably stable and did not show any blinking, as exemplified by the time trace of a single NC presented in the inset of Fig. 1(a). Shot-noise-limited intensity fluctuations were measured over minutes of acquisition times. Figure 1(a) shows the PL spectrum of this NC recorded over one minute at a weak $\mathrm{cw}$ excitation intensity of $60 \mathrm{~W} / \mathrm{cm}^{2}$. Interestingly, the spectrum displays two peaks $0.7 \mathrm{meV}$ apart around $2.027 \mathrm{eV}$ with resolution-limited linewidths $(\sim 300 \mu \mathrm{eV})$. The intensities of the peaks have a linear dependence on the excitation power, excluding any biexcitonic origin for this structure. A similar spectral structure was found for tens of single NCs. These peaks which are labelled $\mathrm{A}$ and $\mathrm{F}$ are attributed to the bright and the dark exciton radiative recombination ZPLs, respectively. Indeed, the peak separation is in agreement with the energy splitting of $\sim 1 \mathrm{meV}$ between $|A\rangle$ and $|F\rangle$ expected from the theoretical analysis of the exciton fine structure for $4 \mathrm{~nm}$ NCs [11]. The peak around $2.00 \mathrm{eV}$, which is $26.6 \mathrm{meV}$ red-shifted from the main peak F, corresponds to the longitudinal optical (LO) phonon replica of the $|F\rangle$ state ZPL [17]. One can also distinguish a weaker structure denoted Ac, $0.8 \mathrm{meV}$ red-shifted from F. We attribute this feature to a confined acoustic phonon sideband due to electron coupling to the acoustic radial breathing mode of the NC [19, 20].

In order to confirm the attribution of the emission peaks $\mathrm{A}$ and $\mathrm{F}$, we studied their temperature evolution on the same $\mathrm{NC}$, as shown in Fig. 1(b). Comparing spectra from $2 \mathrm{~K}$ to $10 \mathrm{~K}$, one can clearly see that the relative intensity of the peak A increases with temperature, 
in agreement with the picture of a phonon mediated thermal mixing between the bright and the dark excitons. The broadening of the peaks is attributed to the population of acoustic phonon modes that rises with temperature, washing out the two ZPLs above $15 \mathrm{~K}$.

The striking observation of the two ZPLs in the PL spectrum points to a reduced spinflip relaxation rate in these relatively large NCs, in contrast to small NCs where only a single ZPL (attributed to the $|F\rangle$ state, the thermal population of the $|A\rangle$ state being negligible) is observed. As a typical example, Fig. 1(c) shows the emission spectrum of a $\sim 2.2 \mathrm{~nm}$ radius single $\mathrm{NC}$ at $2 \mathrm{~K}$. In this case, the integrated intensity of the ZPL is weaker than that of the acoustic phonon sideband, supporting the assumption that the radiative recombination from this state occurs preferentially with emission of phonons. Yet the origin of the dark exciton purely radiative recombination is still an open question. Semiempirical pseudopotential calculations [21] show that this recombination can be due to a surface-induced mixing of dark and bright exciton states. However, spectroscopic investigations led on CdSe NCs with different coating shells argue against the involvements of surface states, and suggest a mechanism intrinsic to the CdSe NCs [22]. Beyond the effective mass approximation, semiempirical tight-binding calculations that incorporate spin-orbit interactions [23] provide an explanation for the finite lifetime of the dark exciton and for its decrease with the NC radius $[13,22]$. Other recombination mechanisms may involve spin-flip transitions of the $|F\rangle$ state induced by paramagnetic defects in the lattice [11], or by the hyperfine interaction with unpolarized nuclear spins [24].

We also performed ultra-high resolution resonant photoluminescence excitation (RPLE) of the two lowest energy ZPLs of a single NC at 2 K. PLE of upper levels of the bandedge exciton fine structure has already been measured on individual NCs and led to linewidths of a few meV [25]. Our new method consists in sweeping the frequency of a cw single mode tunable dye laser (spectral resolution $\sim 4 \mathrm{neV}$ ) around the central frequencies of the A and F ZPLs, while collecting the red-shifted luminescence of the LO mode through a long pass filter on an avalanche photodiode. The relative weight of the LO-phonon line compared to the ZPL is of the order of $10 \%$, giving a count rate of thousands per second sufficiently high to record R-PLE spectra. The R-PLE lines of a single NC are displayed in Fig. 2 (a-c) at a very low excitation intensity of about $1 \mathrm{~W} / \mathrm{cm}^{2}$, (well below saturation, the signal being linear with intensity). In the case of single scan spectra [see Fig. 2(b,c)] recorded on a time scale of $100 \mathrm{~ms}$, which corresponds to the passage time through resonance, the lines are extremely sharp and display linewidths of the order of $10 \mu \mathrm{eV}$. Such linewidths rule out 
any correspondence with a phonon band for these lines [25], and therefore supports their attribution to purely electronic lines. These spectra display the narrowest homogeneous lines ever recorded on individual CdSe NCs, setting a lower bound of 100 ps for the optical coherence lifetime of the two lowest energy bright and dark excitons over a time scale as long as $100 \mathrm{~ms}$. Dephasing times of the same order of magnitude, but over a time scale of $200 \mu \mathrm{s}$, were recently reported by Coolen et al. using a newly developed photon-correlation Fourier spectroscopy technique [26]. Even if the NCs used in our studies seem to present an efficient surface passivation, R-PLE spectra recorded on several frequency sweeps display a broadening with the number of scans $(\sim 30 \mu \mathrm{eV}$, after 10 scans) [Fig. 2 (a)] due to residual spectral diffusion, i.e. random spectral shifts of the transition frequencies due to fluctuations in the local nano-environment.

In order to explain our observation of the lowest energy ZPL doublet, we need to determine the relaxation rates involved in the emission dynamics. Therefore, we studied the temperature dependent PL decay of many single NCs. Figure 3(b) shows the PL decay of a single $\mathrm{NC}$ for various temperatures ranging from $2 \mathrm{~K}$ to $105 \mathrm{~K}$. At temperatures below $7 \mathrm{~K}$, the decay shows a biexponential behavior with a fast decay occurring within few ns and a much slower one with a lifetime $\Gamma_{L}^{-1}$ in the $200 \mathrm{~ns}$ range. At higher temperatures $(>10 \mathrm{~K}), \Gamma_{L}^{-1}$ shortens and the fast component vanishes completely, as previously reported [14]. We explain the PL behavior with a three-level model illustrated in Fig. 3(a). It takes into account the zeroexciton ground level, $|G\rangle$, and the two lowest states of the band-edge exciton fine structure, $|A\rangle$ and $|F\rangle$, with respective radiative rates $\Gamma_{A}$ and $\Gamma_{F}$. Thermal mixing between these excitonic states occurs via emission and absorption of acoustic phonons from a mode whose energy $\Delta E$ matches the energy splitting between $|A\rangle$ and $|F\rangle$ states. The $|A\rangle \rightarrow|F\rangle$ and $|F\rangle \rightarrow|A\rangle$ rates are respectively $\gamma_{0}\left(N_{B}+1\right)$ and $\gamma_{0} N_{B}$, where $\gamma_{0}$ is the zero-temperature relaxation rate of the $|A\rangle \rightarrow|F\rangle$ transition and $N_{B}=1 /\left[\exp \left(\Delta E / k_{B} T\right)-1\right]$ is the Bose-Einstein phonon number at temperature $T$. In order to obtain a sufficient counting rate from a single $\mathrm{NC}$, a relatively high laser repetition rate $(2.5 \mathrm{MHz})$ is needed to record the PL decays. As a consequence, the excitation period is not much longer than the lifetime at low temperature. A contribution from a multiexcitonic recombination to the short time decay component cannot be completely excluded, even if the excitation intensity was kept low enough to ensure a mean number of exciton per pulse smaller than one. We therefore used the temperature 
dependence of the long time decay component and PL spectra to determine the relaxation rates. The decay rate of the long time component is deduced from the solutions of rate equations for the excitonic states populations:

$$
\Gamma_{L}=\frac{1}{2}\left\{\Gamma_{A}+\Gamma_{F}+\gamma_{0} \operatorname{cotanh}\left(\frac{\Delta E}{2 k_{B} T}\right)-\sqrt{\left(\Gamma_{A}-\Gamma_{F}+\gamma_{0}\right)^{2}+\gamma_{0}^{2} \sinh ^{-2}\left(\frac{\Delta E}{2 k_{B} T}\right)}\right\}
$$

Figures 3(b) and 3(c) show the temperature dependence of $\Gamma_{L}$ for the NC. It is well reproduced by the expression (1), using only $\gamma_{0}$ and $\Gamma_{A}$ as fitting parameters. Indeed, the parameters $\Delta E=1.4 \mathrm{meV}$ and $\Gamma_{F}=3.7 \times 10^{-3} \mathrm{~ns}^{-1}$ are respectively deduced from the energy difference between the two ZPLs on the PL spectrum [see inset of Fig. 3(c)], and the long time component of the decay in the very low temperature regime $\left(2 \mathrm{~K}, k_{B} T<<E\right)$. From the fit we obtain the bright state decay rate $\Gamma_{A} \sim 0.16 \mathrm{~ns}^{-1}$, and $\gamma_{0}=0.87 \mathrm{~ns}^{-1}$. This weak spin-flip relaxation rate suggests the existence of a phonon bottleneck effect [27-30] for large NCs where the energy splitting $\Delta E$ is in the meV range, and explains the observation of the ZPLs of both $|A\rangle$ and $|F\rangle$ states in the PL spectrum of the NC. Furthermore, the relative weight of the emission rate from the state $|A\rangle$ can be estimated within the frame of our three-level model. Assuming that the two lowest excitonic levels have identical quantum yields and are equally populated from the high energy continuum states [14, 31], this weight is $\Gamma_{A} / 2\left(\Gamma_{A}+\gamma_{0}\right)$ in the low temperature regime. Using the relaxation rates deduced from the PL decay analysis, we obtain a weight of $\sim 8 \times 10^{-2}$, consistent with that deduced from the PL spectrum.

An interesting observation concerns the relative weight of LO-phonon sidebands with respect to the ZPLs in the PL spectra. PL spectra of single NCs, such as those presented in Fig. 1(a) or Fig. 4(a), do not display LO-phonon replica of the bright state ZPL at $2 \mathrm{~K}$, whereas the weight of the LO-phonon band represents $\sim 20 \%$ of the total emission from the dark $|F\rangle$ state. As we raise the temperature from $2 \mathrm{~K}$ to $10 \mathrm{~K}$, the weight of the LO-phonon band significantly drops with respect to the high energy structure (which includes $\mathrm{A}$ and $\mathrm{F}$ ZPLs and Ac) [see inset of Fig. 4 (a)]. A similar behavior has been reported on ensemble measurements using fluorescence line narrowing spectroscopy [32]. Our thermal mixing model now explains this observation as follows: An increase of temperature will lead to an 
increase of the $|A\rangle$ state ZPL and therefore to a decrease of the LO-phonon band relative weight. Most of the studied NCs displayed a less efficient LO-phonon coupling for the bright exciton than the dark. Note that we found few NCs show similar LO-phonon couplings for both states, as exemplified in Fig. 4 (b). As expected from the model, the weight of the LOphonon replica does not depend on temperature [see inset of Fig. 4 (b)] in this case.

In summary, we observed in the low temperature emission spectra of single $\mathrm{CdSe} / \mathrm{ZnS}$ NCs two ZPLs attributed to the radiative recombinations from the two lowest levels of the band-edge exciton fine structure. This observation points to a size dependence of the brightto-dark spin-flip rate. From the analysis of the temperature dependent PL decay rate and the PL spectrum with a three-level model, we can deduce the bright exciton radiative rate as well as the bright-to-dark spin-flip rate for a single NC. We also performed for the first time ultrahigh resolution R-PLE of the two lowest energy ZPLs for a single NC at $2 \mathrm{~K}$. This experiment sets a lower bound of $100 \mathrm{ps}$ for the optical coherence lifetime of the two lowest energy bright and dark excitons over a time scale as long as $100 \mathrm{~ms}$. Further investigations will aim at determining the dependence of the exciton relaxation rates on the NC size or applied magnetic fields.

We thank Alexander Efros for fruitful discussion and Jonah Shaver for careful reading of the manuscript. This research was funded by the Région Aquitaine, the Agence Nationale pour la Recherche (ANR) and the French Ministry for Education and Research (MESR).

\section{References}

[1] D. J. L. A.L. Efros, and L. Tsybeskov, Semiconductor Nanocrystals: From Basic Principles to Applications (Kluwer Academic/Plenum Publishers, New York, 2003).

[2] V. L. Colvin, M. C. Schlamp, and A. P. Alivisatos, Nature 370, 354 (1994).

[3] B. O. Dabbousi et al., Applied Physics Letters 66, 1316 (1995).

[4] M. C. Schlamp, X. G. Peng, and A. P. Alivisatos, Journal of Applied Physics 82, 5837 (1997).

[5] V. I. Klimov et al., Science 290, 314 (2000).

[6] M. Bruchez et al., Science 281, 2013 (1998).

[7] X. Michalet et al., Science 307, 538 (2005).

[8] A. P. Alivisatos, Science 271, 933 (1996).

[9] V. I. Klimov, Semiconductor and Metal Nanocrystals:Synthesis and Electronic and Optical Properties (CRC Press, 2003).

[10] M. Nirmal et al., Physical Review Letters 75, 3728 (1995).

[11] A. L. Efros et al., Physical Review B 54, 4843 (1996).

[12] E. Johnston-Halperin et al., Physical Review B 63 (2001). 
[13] S. A. Crooker et al., Applied Physics Letters 82, 2793 (2003).

[14] O. Labeau, P. Tamarat, and B. Lounis, Physical Review Letters 90 (2003).

[15] N. Le Thomas et al., Phys Rev Lett 94, 016803 (2005).

[16] M. Chamarro et al., Physical Review B 53, 1336 (1996).

[17] S. A. Empedocles, D. J. Norris, and M. G. Bawendi, Physical Review Letters 77, 3873 (1996).

[18] Y. M. Shen et al., Physical Review B 76 (2007).

[19] G. Chilla et al., Physical Review Letters 100 (2008).

[20] M. J. Fernee et al., Journal of Physical Chemistry C 112, 1878 (2008).

[21] M. Califano, A. Franceschetti, and A. Zunger, Nano Letters 5, 2360 (2005).

[22] C. D. Donega, M. Bode, and A. Meijerink, Physical Review B 74 (2006).

[23] K. Leung, S. Pokrant, and K. B. Whaley, Physical Review B 57, 12291 (1998).

[24] J. A. Gupta et al., Physical Review B 66 (2002).

[25] H. Htoon, P. J. Cox, and V. I. Klimov, Physical Review Letters 93 (2004).

[26] L. Coolen et al., Physical Review Letters 100 (2008).

[27] U. Bockelmann, Physical Review B 50, 17271 (1994).

[28] E. A. Muljarov, T. Takagahara, and R. Zimmermann, Phys Rev Lett 95, 177405 (2005).

[29] J. I. Climente et al., Physical Review B 74 (2006).

[30] T. Nakaoka et al., Physical Review B 74 (2006).

[31] A. L. Efros, Physical Review B 46, 7448 (1992).

[32] M. Nirmal, C. B. Murray, and M. G. Bawendi, Physical Review B 50, 2293 (1994). 


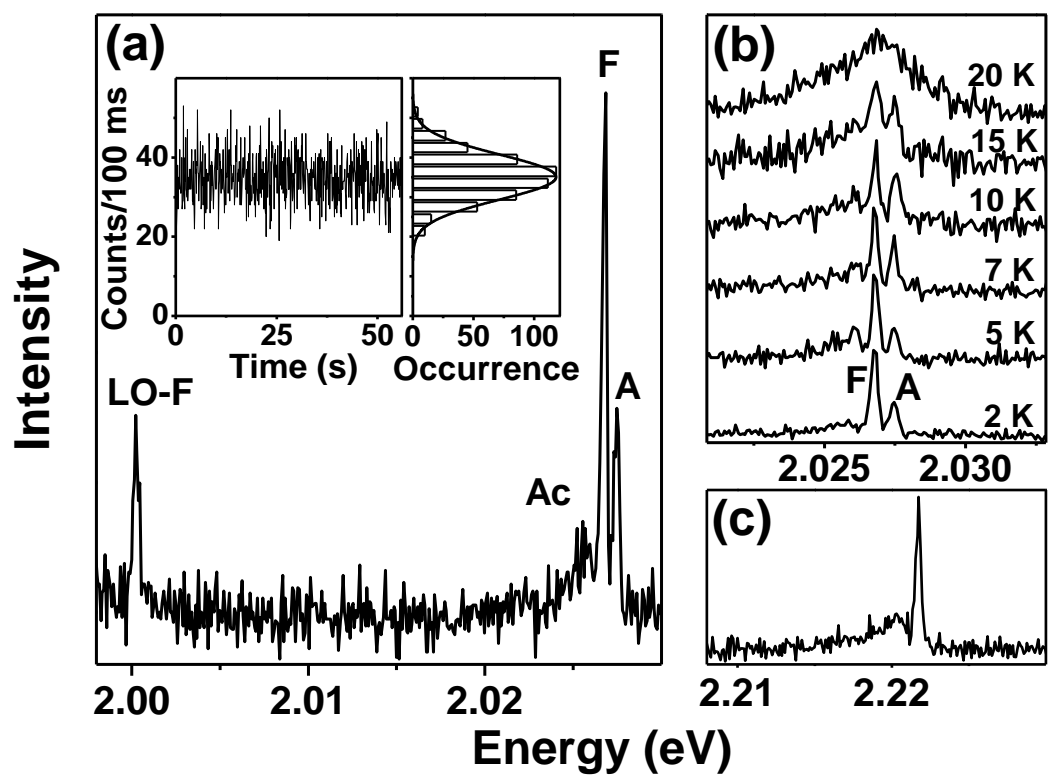

FIG. 1. (a) PL spectrum at $2 \mathrm{~K}$ of a single NC (radius $\sim 4 \mathrm{~nm}$ ), recorded with an integration time of $60 \mathrm{~s}$ and at an excitation intensity of $60 \mathrm{~W} / \mathrm{cm}^{2}$. The peaks labelled $\mathrm{F}$ and $\mathrm{A}$ are attributed to the ZPLs of the dark and the bright excitons respectively (see the text). The peak LO-F is a LO-phonon replica of the peak F. The Ac structure is a confined acoustic phonon sideband of the peak F. The inset shows the time trace of the integrated PL intensity recorded simultaneously with the PL spectrum for this NC. The distribution of the photon counts per bin $(100 \mathrm{~ms})$ shows that the intensity fluctuations are shot-noise limited. (b) A Zoom of the PL spectrum shown in Fig 1(a), recorded at various temperatures ranging from $2 \mathrm{~K}$ to $20 \mathrm{~K}$. (c) A typical PL spectrum at $2 \mathrm{~K}$ for a $\sim 2.2 \mathrm{~nm}$ radius individual NC. The presence of a single sharp line is attributed to the $|F\rangle$ state ZPL. 


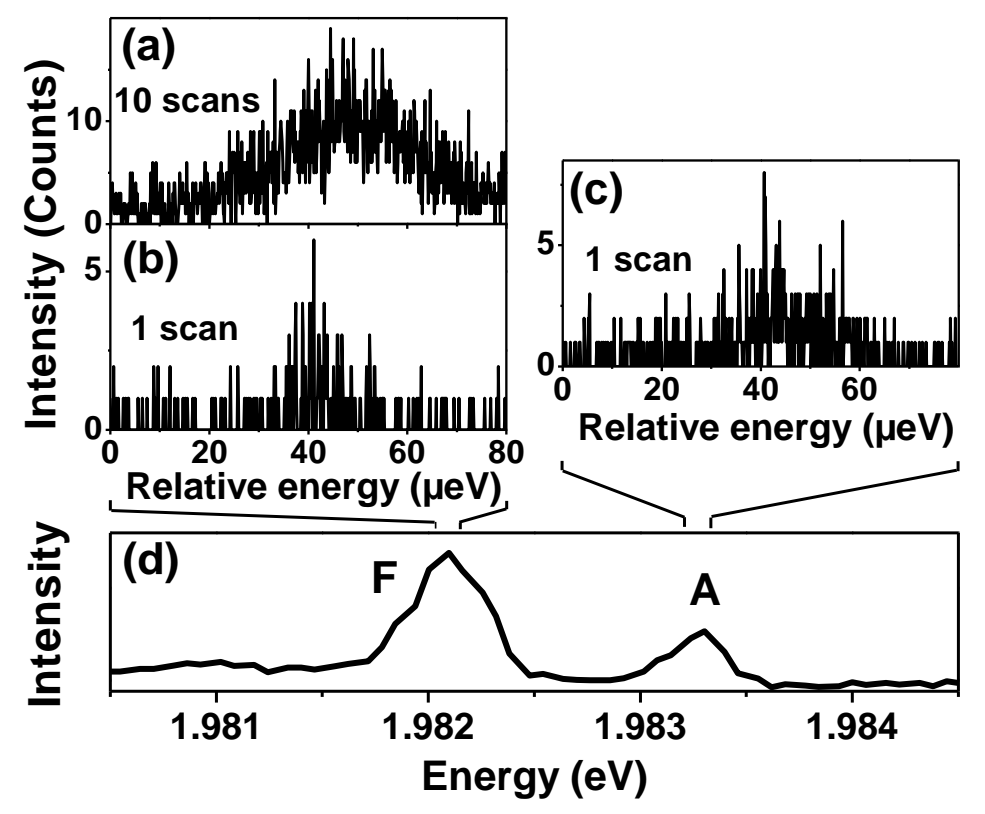

FIG. 2. R-PLE and PL spectra of a single NC at 2 K. (a) R-PLE spectrum of the $|F\rangle$ state ZPL, recorded on ten frequency scans at an excitation intensity of $1 \mathrm{~W} / \mathrm{cm}^{2}$. The duration of one scan is 1 s. (b) R-PLE spectrum of the $|F\rangle$ state ZPL recorded on a single scan, showing a linewidth of $\sim 10 \mu \mathrm{eV}$. (c) R-PLE spectrum of the $|A\rangle$ state ZPL recorded on a single sweep. (d) A zoom of the PL spectrum on the ZPL doublet. 

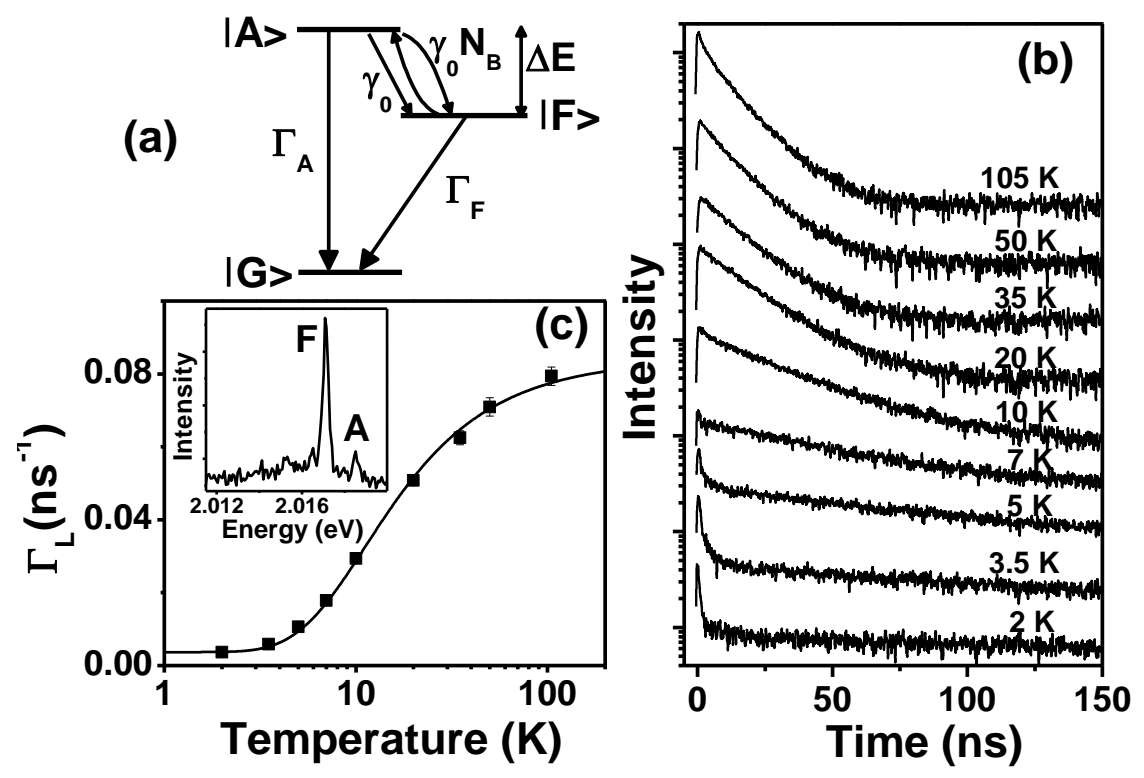

FIG. 3. (a) The three-states model. $|G\rangle$ is the ground state, $|A\rangle$ and $|F\rangle$ are the lowest bright and dark states with radiative relaxation rates $\Gamma_{A}$ and $\Gamma_{F}$ respectively. $\Delta E$ is the energy splitting between these states. $\gamma_{0}$ is the zero-temperature spin-flip rate from $|A\rangle$ to $|F\rangle \cdot \gamma_{0} N_{B}$ is the thermal mixing rate between $|A\rangle$ and $|F\rangle$ due to acoustic phonons. (b) Luminescence decay curves of a single $\mathrm{NC}$ at various temperatures (average excitation intensity of 20 $\mathrm{W} / \mathrm{cm}^{2}$, corresponding to less than one exciton prepared per pulse). (c) Temperature dependence of the slow component decay rate $\Gamma_{L}$. The solid line is a fit derived from the three-level model, with the two fitting parameters $\Gamma_{A}$ and $\gamma_{0}$ (see text). The inset shows the PL spectrum of this NC at $2 \mathrm{~K}$ giving the energy splitting between the states $|A\rangle$ and $|F\rangle$. 


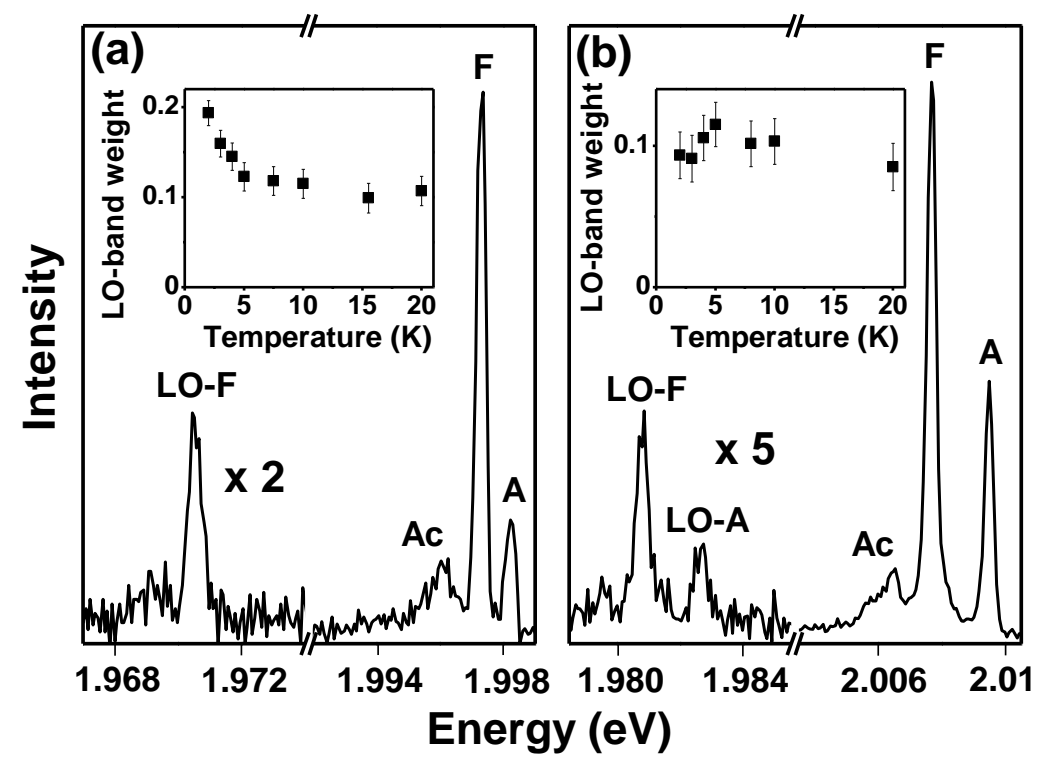

FIG. 4. Emission spectra at $2 \mathrm{~K}$ of two single NCs with different LO-phonon couplings. (a) The LO-phonon yield from the dark state is larger than that from the bright state. (b) The LOphonon yields from the dark state and from the bright state are identical. The insets show the weight of the integrated intensity of the LO-phonon structure as a function of temperature. 